\title{
Evaluation of a Ketogenic Diet for Improvement of Neurological Recovery in Individuals with Acute Spinal Cord Injury: study protocol for a randomized controlled trial
}

\author{
Aynur DEMIREL \\ Hacettepe Universitesi \\ Jia LI \\ University of Alabama at Birmingham \\ Casey MORROW \\ The University of Alabama at Birmingham

\section{Stephen BARNES} \\ The University of Alabama at Birmingham \\ Jan JANSEN \\ University of Alabama at Birmingham

\section{Barbara GOWER} \\ University of Alabama at Birmingham

\section{Keneshia KIRKSEY} \\ University of Alabama at Birmingham

\section{David REDDEN}

The University of Alabama at Birmingham

\section{Ceren Yarar-Fisher ( $\nabla$ cyarar@uab.edu )}

University of Alabama at Birmingham Department of Medicine

\section{Study protocol}

Keywords: Spinal cord injury; Diet, ketogenic; Proteomics; Nutrition Therapy; High-protein diet; Lowcarbohydrate diet

Posted Date: February 12th, 2020

DOI: https://doi.org/10.21203/rs.2.23366/v1

License: (c) (i) This work is licensed under a Creative Commons Attribution 4.0 International License. Read Full License 
Version of Record: A version of this preprint was published at Trials on May 4th, 2020. See the published version at https://doi.org/10.1186/s13063-020-04273-7. 


\section{Abstract}

Background: Therapies that significantly improve the neurological and functional recovery of individuals with spinal cord injury (SCI) are still urgently needed. The ketogenic diet (KD) has been shown to improve forelimb motor function in a rat model of $\mathrm{SCl}$, likely by reducing inflammation and cell death in the spinal cord. Furthermore, our recent pilot study in patients with $\mathrm{SCl}$ showed that, compared with a standard hospital diet (SD), 5 weeks of KD, started during acute care, improved upper extremity motor function and reduced serum levels of a neuroinflammatory blood protein. The primary goals of our proposed study are to 1 ) show the safety and feasibility of administering a KD during acute care for $\mathrm{SCl} ; 2$ ) determine if consuming 5 weeks of a KD significantly improves motor and sensory function, functional independence, and glycemic control; and 3) quantify serum biomarkers that are linked to improvements in neurological recovery and functional independence via targeted proteomics.

Methods/Design: In a single-masked, longitudinal, randomized parallel controlled study design, 60 participants with acute $\mathrm{SCl}$ will be randomly assigned to $\mathrm{KD}$ or SD in a 1:1 ratio. We intend to recruit 24 participants for each group with traumatic $\mathrm{SCl}$ (C5-T12, American Spinal Injury Association impairment scale $A-C$, and aged 18-60 years). Patients will be taken to acute care after their injury. Neurological and functional examinations, resting energy expenditure, blood, urine, stool collection and protein analyses related to neuro recovery will be done within $72 \mathrm{~h}$ of injury, as baseline measures. Patients received five weeks of KD or SD according to their allocation. The KD is a high-fat, low-carbohydrate diet (3:1 ratio of fat: carbohydrate + protein) that includes $~ 75 \%$ total energy as fat, $20 \%$ as protein, and $\sim 5 \%$ as carbohydrate and fiber. The SD includes $\sim 45-50 \%$ total energy as carbohydrate and fiber, $\sim 30 \%$ total energy as fat, and $\sim 20 \%$ total energy as protein. All baseline examinations will be repeated at discharge from the hospital at week 5 (discharge measure).

Discussion: The proposed diet intervention is practical and safe and is expected to translate into efficacious early nutritional interventions. Intervention with a more neuroprotective diet during acute care of $\mathrm{SCl}$ can be implemented anywhere in the world at low cost and without major regulatory hurdles. Better functional recovery will lead to a better quality of life and long-term health outcomes in individuals with $\mathrm{SCl}$. While this study targets $\mathrm{SCl}$, if successful it has the potential to improve neurological outcomes for individuals with various traumatic injuries such as traumatic brain injury and stroke.

\section{Background}

Despite the advances in understanding the basic neurobiology of spinal cord injury (SCl), the development of therapeutic strategies for the acutely injured spinal cord has been agonizingly slow; thus, $\mathrm{SCl}$ remains a significant cause of disability and mortality. Spinal cord injury in humans not only leads to the well-recognized loss of motor and sensory function, but also the less-studied multiple secondary complications (i.e., bladder and bowel dysfunction, metabolic disorders, and cardiovascular disease) caused by extreme physical inactivity due to paralysis (1-3). These secondary complications of SCl contribute to the greatly reduced lifespan of these patients, which is rarely emphasized. While the field 
has made large strides in improving the survival of individuals with SCl in the acute 2-year phase after injury (40\% reduction in mortality), there have been no gains in lifespan in the chronic situation over the last 20 years (4). Therefore, individuals with SCI who survive beyond the first 2 years after injury still die, on average, 13-25 years earlier than able-bodied people, depending on the level and severity of injury (46 ). The current paradigm holds that better neurological recovery during the acute period will lead to a longer, more active and independent life for individuals with SCl. In addition, individuals with improved neurological recovery are expected to better manage problems such as spasticity, weight gain, and chronic pain and to be less likely to experience feelings of anxiety, loneliness, and depression.

After the initial trauma of $\mathrm{SCl}$, cell death and tissue loss continue for several weeks (7)-a window in which one could effectively intervene with neuroprotective strategies. During this time, an escalating cycle of glutamate excitotoxicity, inflammation, and neuronal apoptosis leads to progressive degeneration in the spinal cord (8). Currently, the only approach taken to restrict secondary damage after SCl is surgical decompression and the administration of methylprednisolone. However, the recommendations to use methylprednisolone have fluctuated from "standard of care" to "optional" to "not recommended" and currently back to "optional" in the US and Canada since it has been shown to offer merely marginal benefit in select patient groups and increased risk of major complications, in particular, gastrointestinal ulcers/bleeding during the acute stage of $\mathrm{SCl}(7)$. In addition, several trials of neuroprotective treatments given in the acute phase, after $\mathrm{SCl}$, have failed (9), while other approaches such as therapeutic hypothermia and administration of drugs targeting inflammation or glutamate excitotoxicity, for example, are still under investigation (8) and not standard of care. This situation highlights the urgent need for new approaches for preventing secondary damage during the acute phase of $\mathrm{SCl}$.

Emerging evidence suggests that acute-phase hyperglycemia is a critical indicator of poor functional outcomes of $\mathrm{SCl}(10,11)$. The presence of hyperglycemia $(\geq 126 \mathrm{mg} / \mathrm{dl})$ on hospital admission (irrespective of past diabetes mellitus history) was found to be strongly associated with a lower probability of improvement in motor and sensory functions. Changes to diet might be the least invasive approach to recovery after neurotrauma. A wide variety of evidence suggests that the ketogenic diet (KD) could have beneficial disease-modifying effects in a broad range of neurological disorders (12-16). The $\mathrm{KD}$ is composed of $80-90 \%$ fat, with carbohydrate and protein constituting the remainder of the intake. Energy is largely derived from the utilization of dietary fat. These fats are converted in the liver to ketone bodies $\beta$-hydroxybutyrate, acetoacetate, and acetone, which provide the brain with an alternative energy source to glucose. The ability of a KD to ameliorate the diabetic state and help stabilize hyperglycemia has been repeatedly shown in human studies $(17,18)$. In addition, a study by Streijger et al. $(2013)$ showed the critical role of ketones in neuroprotection by starting a KD four hours after cervical hemicontusion in rats with $\mathrm{SCI}(19)$. Consistent with the published effects of a KD in rat models of $\mathrm{SCl}$ and in humans with hyperglycemia, our randomized pilot feasibility trial (20) showed that, compared with a standard hospital diet (SD), five weeks of KD improved upper extremity motor scores (Fig. 1).

Although the exact mechanism by which the KD provides neuroprotection is not fully understood, three main effects on cellular energetics, mitochondria function, and inflammation have been suggested to 
play a key role $(21,22)$. Consequently, KD may be optimal for maintaining a normal glycemic state, promoting inflammation regulation, and rescuing mitochondrial function after $\mathrm{SCl}$. Therefore, in this clinical trial, our purposes are to (1) show safety and feasibility to administer a KD in the acute stages of $\mathrm{SCl}$, (2) determine if 5 weeks of KD vs. SD significantly improves motor and sensory function, functional independence, glycemic control and microbiome composition in patients with acute SCl, and (3) quantify serum biomarkers that are linked to improvements in neurological recovery and functional independence via targeted proteomics.

\section{Research Methods/design}

\section{Study design and participants}

In a single-masked, longitudinal, randomized parallel controlled study design, eligible participants who provide informed consent ( $\mathrm{PI}$, and assigned study coordinator will obtain the consent) are randomly assigned into a KD intervention group or an SD control group in a 1:1 ratio. Randomization is performed using the block randomization method, with a block size of four by the study statistician. A randomization list is generated, and the random assignments are placed into closed envelopes. Primary Investigator (PI) is responsible for participant recruitment and trial coordination. The prospective participant or legally authorized representative (if the patient is under mechanical ventilation) will provide consent. All nurses performing blood draws, and core facilities and staff (research technicians, students, and trainees) analyzing the primary outcomes are blinded to group assignment. A Standard Protocol Items: Recommendations for Interventional Trials (SPIRIT) schedule is presented in Fig. 2 and the SPIRIT checklist is shown as Additional file 1. Outcome measures are performed at three time points: at baseline within 72 hours of injury, before discharge from the acute care unit to the rehabilitation hospital, and at discharge from the rehabilitation hospital. Neurological examination via International Standards for Neurological Classification of SCI (ISNCSCI), commonly referred to as the American Spinal Injury Association Impairment Scale (AIS), electrical perception thresholds (EPTs)(23), functional examinations via Spinal Cord Independence Measure $(\operatorname{SCIM})(24,25)$, resting energy expenditure (REE), and blood collection (for metabolic, targeted proteomics, and safety outcomes) are done within $72 \mathrm{~h}$ of injury, prior to starting enteral feeding (baseline measure). Following acute care, patients are transferred to the University of Alabama at Birmingham (UAB) Spain Rehabilitation Center (SRC) for in-patient rehabilitation care with an average length of stay in the rehabilitation care of 20 days for patients with acute $\mathrm{SCl}$. Accordingly, subsequent examinations are done prior to transfer to the SRC (rehabilitation measure) and discharge from the SRC at week 5 (discharge measure). A summary of the data collection schedule for the outcomes is shown in Fig. 2, and the flow diagram for the overall study design is shown in Fig. 3 .

The study is currently being conducted at the UAB Center for Clinical and Translational Science (CCTS), UAB Diabetes Research Center Human Physiology Core, UAB Nutrition and Obesity Research Center Metabolism Core, UAB Microbiome Core, and UAB Targeted Metabolomics and Proteomics Laboratory. Participants are considered eligible if they meet the following eligibility criteria: 1 ) are between the ages of 18 and 60 years; 2) have a diagnosis of traumatic SCl at the cervical or thoracic level (C5-T12); and 3) 
are classified as A (sensorimotor complete), B (sensory incomplete SCl: participants with sensory perception to $S 4$ to $S 5$, but no motor function preserved more than 3 segments below the cervical neurological level of injury), or $\mathrm{C}$ (nonfunctional motor incomplete: sensory and motor function are partially preserved below the neurological level of injury, but more than half the key muscles, below the neurological level of injury, have a muscle grade strength of $(<3 / 5)$ on AIS. The exclusion criteria include 1) patients with suspected traumatic central cord syndrome, because of the expected substantial spontaneous neurorecovery (26); 2) pregnant women; 3 ) neurological (other than SCl), vascular, or cardiometabolic problems (e.g., hypertension or diabetes) that may limit function and interfere with testing procedures; 4) evidence of renal insufficiency or liver disease by history, physical examination, and laboratory tests; 5) underlying pulmonary diseases; and 6) evidence of food allergies limiting consumption of a KD. Because of the differences in metabolic responses and nutritional needs among adults and children in the acute stages of traumatic injury, we only focus on adults with acute $\mathrm{SCl}$ to determine the effects of KD on neurological recovery in this study; children (age $<18$ years) are not eligible for this study.

The study population is expected to be representative of the demographics of the SCl patient population. Based on 2018 Spinal Cord Injury Facts and Figures, 78\% of SCls reported to the national database have occurred among males (27). Therefore, we anticipate that among those who participate in this study, $78 \%$ is male and $22 \%$ is female. Spinal cord injury is more common in non-Hispanic whites $(60.6 \%)$, followed by $21.9 \%$ non-Hispanic black, $12.8 \%$ Hispanic origin, $0.7 \%$ Native American, $2.7 \%$ Asian, and $1.3 \%$ other; therefore, we expect that more non-Hispanic whites, as compared with other racial or ethnic groups, participate in this study.

\section{Recruitment}

Participants are recruited from the UAB Hospital Trauma, Burns, and Surgical Critical Care (Acute Care) Unit. Screening for the target participant pool is conducted by the UAB Emergency Medicine Research Assistance (EMRA) Program. Participants are compensated $\$ 100$ for their time.

\section{Adequacy of the potential participant pool}

The primary duty of EMRA is to maintain a presence in the University Hospital Emergency Department on a $24 \times 7$ basis. This program provides the research infrastructure necessary to carry out screening and enrollment in numerous clinical trials in the Emergency Department, which treats 100,000 people per year. The Research Assistants assist the main research study team in determining patient eligibility by confirming with the existing clinical staff the enrollment inclusion and exclusion criteria. They also notify key research personnel of study enrollment and provide information to the physicians regarding study status, eligibility requirements, and general protocol information.

\section{Interventions/groups}

\section{The Ketogenic Diet (KD) Group}


This diet is a high-fat, low-carbohydrate diet (3:1 ratio of fat: carbohydrate + protein) that includes 75\% total energy as fat, $\sim 20 \%$ as protein, and $\sim 5 \%$ as carbohydrate and fiber. Dietary fat sources focus on animal and vegetable (for vegetarians) fats. Dietary fat resources for vegan participants include nuts, seeds, avocados, and coconut oil. Dietary protein sources include animal, plant, and nut and seed proteins, and dietary carbohydrate resources include vegetarian and vegan sources. KetoCal ${ }^{\circledR}, a$ nutritionally complete, ready-to-feed ketogenic formula in a 3:1 ratio (fat: carbohydrate + protein), is provided by Nutricia for the patients who receive enteral nutrition. Nutricia is a global health company that has been the main provider of ketogenic diets for clinical trials, hospitals, health care professionals, and communities around the world. Patients who are able to swallow receive liquid or solid high-fat meals that are prepared by UAB research kitchen, with the overall energy amount determined according to REE assessed via indirect calorimetry and multiplied by an activity factor. Resting energy expenditure is measured after a 12-h fast.

\section{The Standard Diet (SD) Group}

Standard enteral tube feeding includes $\sim 45-50 \%$ total energy as carbohydrate and fiber, $\sim 30 \%$ total energy as fat, and $\sim 20 \%$ total energy as protein. When patients are able to receive liquid or solid feeding, they are expected to consume $\sim 30 \%$ total energy as fat, $\sim 25 \%$ as protein, and $\sim 45 \%$ as carbohydrate based on our preliminary results. Food is provided by UAB's main hospital kitchen, and patients are allowed to choose options from the standard hospital menu, with the overall energy amount determined according to REE assessed via indirect calorimetry and multiplied by an activity factor.

Resting Energy Expenditure (REE) Measure: REE measurements are performed in the patient's hospital room. Participants lie supine on a comfortable bed, with the head enclosed in a plexiglass canopy. After resting for $15 \mathrm{~min}$, REE is measured for 30 min with a portable computerized, open-circuit, indirect calorimetry system with a ventilated canopy (Vmax ENCORE 29 N Systems, SensorMedics Corporation, Yorba Linda, CA). Oxygen uptake (VO2) and carbon dioxide production (CO2) are measured continuously and values are averaged at 1-min intervals. Resting energy expenditure is calculated from the VO2 and CO2 data. The last 20 min of measurement is used for analysis.

\section{In-patient Rehabilitation Care}

Patients in both groups undergo an intensive rehabilitation program for about 5 weeks. Therapy is offered 5 days (total of $15 \mathrm{~h}$ ) per week. The standard of care for both groups includes: respiration therapy, bed mobility, transfers, wheelchair mobility skills, bowel and bladder management, tone and spasticity management, and skills for performing other activities of daily living. Rehabilitation protocols and treatment hours are recorded for each patient. The average duration of stay in the SRC is 30 days for individuals with tetraplegia (injury level C4-C7) and 21 days for individuals with paraplegia (injury level T1-S5).

\section{Outcome Measures And Analysis}


The primary outcomes are as follows: 1) safety: biomarkers of systemic inflammation (C-reactive protein); lipids (total cholesterol, high-density lipoprotein (HDL), low-density lipoprotein (LDL), and triglycerides); sodium; liver enzymes (alanine aminotransferase (ALT), aspartate transaminase (AST), alkaline phosphatase (ALP), albumin, bilirubin, total protein, nutritional ketosis (beta-hydroxybutyrate) and glycemic status (fasting blood glucose and insulin); 2) neurological recovery (AIS) motor and sensory scores and electrical perceptual thresholds); 3) functional independence (SCIM); 4) microbiome composition; and 5) serum biomarkers (targeted proteomics).

In compliance with goals of the National Institute of Neurological Disorders and Stroke (NINDS) Common Data Elements (CDE) initiative, we are utilizing portions of the SCI CDE that are expected to increase the efficiency and effectiveness of our clinical research study and diet intervention, increase data quality, and facilitate data sharing and comparisons across studies. For data analyses and results reporting, we adhere to the guidelines for reporting results using the International Spinal Cord Core Data Set (28).

\section{Minimally acceptable intervention adherence rates}

Adherence to diet will be closely monitored by the study team by measuring serum beta-hydroxybutyrate levels once a week. Blood concentration of 0.5-3 mM (nutritional ketosis) will be considered safe and acceptable. Adherence will also be assessed using food records. The food record will be a simple check list to indicate that supplied foods were consumed, with space to add the quantity of consumption and any deviations from the protocol. In addition, the study dietician will communicate directly with each participant at least weekly to improve adherence.

\section{Clinical procedures to measure safety}

Fasting blood samples are collected to determine the levels of biomarkers of systemic inflammation, lipids, sodium, liver enzymes, and glycemic status. In addition, blood samples are collected for measurement of blood ketone (beta-hydroxybutyrate) levels three times a week, $2 \mathrm{~h}$ after the first meal of the day, to confirm nutritionally induced ketosis (blood concentration, 0.5-3.0 mM) in the KD group. All serum samples will be obtained in the UAB Hospital Acute Care Unit and then stored in freezers within the Pl's (Yarar-Fisher) research laboratory at $-80^{\circ} \mathrm{C}$ until analyzed by core personnel. Levels of sodium, betahydroxybutyrate, lipids, C-reactive protein, glucose, and insulin are assessed via enzymatic assays in the Diabetes Research Center Human Physiology Core. The concentration of Low-density lipoprotein is calculated using the Friedewald formula. In addition, levels of liver enzymes are measured via enzymatic assays at the UAB Hospital Outreach Laboratory.

\section{Procedures to measure neurological recovery}

Sensory and motor function: The motor and sensory examinations are performed according to the ISNCSCI standards (29-31) and described by AIS. The scales used for the neurological tests for this proposal were selected because they are standard, widely accepted tools and can be readily administered in the acute $\mathrm{SCl}$ setting. The AIS Motor Index Score is used for measuring motor function. This index uses standard manual muscle testing on a six-point scale (graded 0-5). The maximum motor score for upper 
and lower extremity is 50 . For the sensory examination, each dermatome is tested for both sharp (pinprick) and light-touch sensation and sensory status is graded on a three-point scale. Numerically, the sensory scores total 116 points.

Electrical perception thresholds (EPTs) are recorded according to established standards (23). In brief, electrical stimuli are delivered at $3 \mathrm{~Hz}$ at key points within cervical dermatomes (C4 to C8). Stimulation commences at $0 \mathrm{~mA}$ and progressively increase at $0.1-\mathrm{mA}$ intervals until the participant perceives a stimulus. The stimulus is then turned down until perception is lost. This process is repeated three times to generate an average electrical perception threshold for each dermatome.

\section{Procedures to measure functional independence}

The SCIM is used for measuring functional independence $(24,25)$ and is, at present, the only comprehensive rating scale that measures the ability of patients with $\mathrm{SCl}$ to perform everyday tasks according to their value for the patient. It requires no manual testing and the range of the total score is $0-$ 100. Although one American Spinal Injury Association (ASIA) In-Step certified clinician performs all examinations, inter-rater reliability between the primary examiner and back-up examiners are assessed periodically as a quality assurance measure for consistency and a precautionary measure in the event that a back-up examiner might be required. Examiners are blinded to the diet assignment. Neurological examinations are routine both in the acute and in-patient care (in the SRC) facilities under the supervision of physiatrist.

\section{Procedures to determine gut microbiome composition}

Microbiome collection and analysis are performed using established protocols (18). Briefly, stools samples are collected in ParaPak vials (Meridian Biosciences, Inc; Cincinnati, $\mathrm{OH}$ ) and consequently diluted to $0.1 \mathrm{mg} / \mathrm{ml}$ in Cary-Blair medium for a total volume of $20 \mathrm{~mL}$ with $10 \%$ glycerol (by volume). Aliquots of $5 \mathrm{~mL}$ are stored at $-80^{\circ} \mathrm{C}$ in cryovial tubes until DNA extraction. Stool bacterial DNA is extracted using a Zymo Research Fecal DNA isolation kit (Zymo Research; Irvine, CA) per the manufacturer's instructions. Polymerase chain reaction (PCR) is then used to amplify the V4 region of the $16 S$ rRNA gene. The PCR products are then retrieved after separated on an agarose gel using electrophoresis, excised from the gel, and subsequently purified with a QIAquick Gel Extraction Kit (Qiagen; Germantown, MD). The Illumina MiSeq DNA sequencing platform is used to sequence 250 base paired-end kits.

Bioinformatics analysis. Microbiome analyses will be performed using the Quantitative Insights into Microbial Ecology (QIIME) bioinformatics software and QWRAP program as previously described (18). Briefly, quality assessment and filtering low-quality data will be performed using the FASTQC and FASTX toolset, respectively. A combination of tools within the QIIME suite will be utilized for clustering reads into operational taxonomy units (OTU) (uclust), taxa assignment (RDP classifier using the Greengenes 16S rDNA database), and as necessary, alignment and phylogenetic inference (using PyNAST and FastTree). These procedures will allow us to quantitatively assess the microbiome population down to the genus, if not species level. For comparative analyses, indices of alpha (Chao1, Observed species, Phylogenetic 
distance, Simpson' index, and Shannon's index) and beta diversity (Bray-Curtis dissimilarity, unweighted UniFrac and weighted UniFrac) will be computed (18).

\section{Procedures to quantify serum biomarkers that may be linked to improvements in neurological recovery and functional independence}

\section{Targeted Proteomics}

a) Sample Processing: Serum samples are processed using disposable affinity-depletion cartridges (NorGen Biotek Corp) that remove the major serum proteins albumin, alpha-antitrypsin, transferrin, and haptoglobin (32). Protein samples are evaporated and resuspended in $6 \mathrm{M}$ urea, $100 \mathrm{mM}$ Tris buffer at $10 \mathrm{mg} / \mathrm{mL}$. Aliquots of the affinity-depleted serum are reduced using dithiothreitol $(25 \mathrm{mM})$ at $50{ }^{\circ} \mathrm{C}$ for $30 \mathrm{~min}$ followed by alkylation of free thiol groups with iodoacetamide $(55 \mathrm{mM})$ for $30 \mathrm{~min}$ in the dark at room temperature. Solutions are then diluted 1/10 in double-distilled water ( $\mathrm{ddH} 2 \mathrm{O})$ and overnight digestion is carried out using mass spectrometry (MS)-grade trypsin $(0.2 \mu \mathrm{g} / \mu \mathrm{L})$. The digests are evaporated to dryness in a Speedvac and then resuspended in $50 \mu \mathrm{L}$ of ddH 20 with $0.1 \%$ formic acid.

b) Targeted protein analysis: Peptides representing fibrinogen, extracellular signal-regulated kinase 1/2, $\mathrm{CD} 11 \mathrm{~b} / \mathrm{CD} 18$ integrin receptor, and the epidermal growth factor is selected from either preliminary data (fibrinogen) or using Skyline, a targeted proteomics software tool (33). Predicted product ions from each peptide are used to set up a multiple reaction monitoring liquid chromatography tandem MS (LC-MS/MS) assay carried out on a SCIEX 6500 Qtrap using micro-flow LC. This instrument is very sensitive and is capable of detecting peptides in the amol range. The tryptic peptides are resolved on a $5-50 \%$ linear gradient of acetonitrile in $0.1 \%$ formic acid mobile phase before entering the electrospray ionization (ESI) interface of the mass spectrometer. Synthetic peptides will be used to obtain quantitative data. To obtain absolute quantitative data on the selected peptide(s), 13C/15N-isotopically labeled versions of the peptide(s) will be prepared (34).

c) Protein Library Generation: An aliquot $(5 \mu \mathrm{L})$ of each digest is loaded onto a Nano cHiPLC $200 \mu \mathrm{m} \times$ $0.5 \mathrm{~mm}$ ChromXP C18-CL 3- $\mu \mathrm{m}$ 120-Å reverse-phase trap cartridge (Eksigent, Dublin, CA) at $2 \mu \mathrm{L} / \mathrm{min}$ coupled to an Eksigent $415 \mathrm{LC}$ system autosampler. After washing the cartridge for $10 \mathrm{~min}$ with $0.1 \%$ formic acid in ddH2O, the bound peptides are flushed onto a Nano cHiPLC column $(200 \mu \mathrm{m} \mathrm{ID} \times 15 \mathrm{~cm}$ ChromXP C18-CL $3 \mu \mathrm{m} 120 \AA$ A, Eksigent) with a 100-min linear (5-50\%) acetonitrile gradient in $0.1 \%$ formic acid at $1000 \mathrm{~nL} / \mathrm{min}$ using an Eksigent Nano1D + LC. The column then is washed with $90 \%$ acetonitrile- $0.1 \%$ formic acid for $5 \mathrm{~min}$ and reequilibrated with $5 \%$ acetonitrile- $0.1 \%$ formic acid for 15 min. A SCIEX 5600 TripleTof mass spectrometer(SCIEX, Toronto, Canada) is used to analyze the protein digest. The ionization spray voltage is set at $2300 \mathrm{~V}$ and the declustering potential set at $80 \mathrm{~V}$. The ionization spray and curtain gases are set at $10 \mathrm{psi}$ and $25 \mathrm{psi}$, respectively. The interface heater 
temperature is $120^{\circ} \mathrm{C}$. In each 1.25-s duty cycle, eluted peptides are subjected to a 250-ms time-of-flight survey scan from m/z 400-1250 to determine the top 20 most intense ions for MS/MS analysis. Product ion time-of-flight scans at $50 \mathrm{~ms}$ are carried out to obtain the tandem mass spectra of the selected parent ions over the range from $\mathrm{m} / \mathrm{z} 400-1000$. Spectra is centroided and de-isotoped by Analyst software, version 1.7 TF (SCIEX). A $\beta$-galactosidase trypsin digest is used to establish and confirm the mass accuracy of the mass spectrometer. The MS/MS data is processed to provide protein identifications using an in-house Protein Pilot 4.5 search engine (SCIEX) using the UniProt Homo sapiens protein database and a trypsin digestion parameter. Proteins are included in the SWATH library on the criteria of having at least two peptides detected with a confidence score of $95 \%$ or greater using the Paradigm method imbedded in the Protein Pilot software.

d) SWATH-MS Analysis (35): Individual samples are analyzed by the same nanoLC-ESI-MS/MS method described above. SWATH data are collected in 1.8-s duty cycles - a 200-ms time-of-flight survey scan from $\mathrm{m} / \mathrm{z}$ 400-1250 followed by thirty-two, 50-ms successive tandem mass spectra using $25 \mathrm{~m} / \mathrm{z}$ mass windows. The MS/MS data are de-convoluted and processed using PeakView ${ }^{\mathrm{TM}} 1.2$ with the SWATH Application (SCIEX). This software uses a pre-generated protein identification library to construct individual peptide signatures with fragmentation patterns after analyses for protein identifications. In addition, the areas of the peptide peaks in the MS/MS spectra are used for protein quantification. Identification of proteins associated with the phenotypes being studied will be carried out by univariate (Volcano plots) and multivariate (principal components and partial least squares-discriminant analysis) analyses and associated heat-maps.

\section{Power Calculations}

Power calculations were performed using nQuery Advisor + nTerim 3.0, and assume a two-sided statistical test and a significance level of $5 \%$. We obtained estimates of the standard deviation for the motor score of 23.5 and for the light touch score of 33 (both based on our preliminary data). With a final sample size of 24 participants per group, and also assuming a two-group t-test and the prior assumptions, we have $80 \%$ power to detect between-group differences of 19.5 in the motor score and 27.3 in the light touch score as being statistically significant. With a final sample size of 24 participants per group, and assuming a paired t-test and the prior assumptions, we have $80 \%$ power to detect withingroup changes of 14.1 in the motor score and 19.8 in the light touch score as being statistically significant. All of these differences are approximately at the same levels as those that Kramer et al. (2012) detected as being statistically significant and clinically meaningful (36). We believe that these estimates are conservative since we are performing our primary statistical analyses for between-group and within-group comparisons simultaneously, using statistical methods that are more sophisticated than those that are assumed here. Given our history in $\mathrm{SCl}$ patient recruitment and the large $\mathrm{SCl}$ patient base at the UAB SRC, we fully expect to meet our recruitment goal.

\section{Randomization and Blinding}


Randomization is performed using the block randomization method, with a block size of 4 (Study statistician). A randomization list generated, and the random assignments were placed into closed envelopes. Each study participant opens one of these envelopes to learn of his/her group assignment. Study patients will inevitably know their group assignment, as we are limited by difficulties inadequate masking of the ketogenic diet, which is very restrictive and requires avoidance of usual foods like bread, pasta, rice, potatoes, and a wide variety of fruits. In order to avoid a potential placebo effect or a participant biasing results due to knowledge of their diet, during the consenting process we simply explain that the study is designed to examine the impact of KD and SD upon outcomes. In addition, individuals performing clinical and laboratory tests and the study statistician were blinded to the study interventions.

\section{Data Management}

To protect privacy, each study participant is assigned a unique 4-digit identification number that cannot be traced to any protected health information (PHI). All data forms, participant information, and biological specimens are coded using these ID numbers. No personally identifiable information (PHI) appears on these materials; instead, the keys linking participants' identities to their unique identification numbers are stored separately in a secure software system designed for clinical trials, which meets both HIPAA and 21 CFR Part 11 requirements.

Data Capture, Verification, and Disposition. All study data is managed in a central database using REDCap, a secure web software system designed for clinical trials, which meets both HIPAA and 21 CFR Part 11 requirements. All questionnaires and daily surveys are coded into REDCap and administered electronically. Data from serum assays, resting energy expenditure measurements, and sensory measures via dermatomal somatosensory evoked potentials and electrical perception thresholds are outputted from the respective technical equipment in electronic form and then uploaded directly into the REDCap database. However, data on adverse events that are collected by the medical team are recorded on paper forms, stored in the Clinical Research Unit, and then manually entered into the REDCap database. Neurological data (assessed by ISNCSCI standards) and food intake data are captured on paper forms and double entered into the database.

Paper documents are scanned and saved on UAB's secure network and/or stored in locked cabinets in the research coordinator's office. The $\mathrm{PI}$ and post-doctoral trainee periodically check the database for missing data and document all such data and the reasons for absence. The study statistician also periodically performs a quality check of the database, which includes error checks using expected ranges for data values. After the study has completed, all data quality will be checked by the statistician, $\mathrm{Pl}$, and the postdoctoral trainee in year 3; tasks are divided among the three personnel according to their respective responsibilities. In compliance with UAB Institutional Review Board (IRB) policies, all data and records will be kept for at least 3 years after the study is completed; all PHI for participants will be deleted 3 years after the trial is completed, while the de-identified final dataset will be retained indefinitely and published online for other scientists to benefit from. 


\section{Statistical Analysis}

Statistical analyses for neurological recovery will include comparisons of means of motor and sensory function, functional independence, and serum measurements (e.g., glucose, insulin, ALT, and AST) between the two groups, as well as comparisons within each group of the changes from baseline to rehabilitation to discharge (which should take approximately 5 weeks). Descriptive statistics, including measures of central tendency (sample mean, sample median) and dispersion (variance) will be calculated for each group. For safety outcomes, instead of comparing the two groups to the established standard safety values, we will compare circulating levels of proposed outcomes at the various time points among the two groups. If there are no significant group differences in these outcomes, we will conclude that the KD intervention is safe. We will also calculate $95 \%$ confidence intervals for the mean group differences for these outcomes as an additional check on safety and feasibility. The primary method of analysis will be analysis of covariance (ANCOVA), regressing the 5-week outcomes (safety, motor and sensory recovery, functional independence, and glycemic state) on the randomly assigned diet, baseline values of the measures, and other covariates such as age and sex. Repeated measures ANCOVA will also be used. Statistical assumptions will be assessed using box plots, residual plots, and normal probability plots. Secondary analyses, accounting for all time points, will be conducted using mixed linear models, including repeated measures models. An appropriate structure for the covariance matrix (e.g. the unstructured covariance matrix) will be selected for these models using the final data. When a model term is statistically significant, the Tukey-Kramer multiple comparisons test will be used to determine which specific pair of means are significantly different. This method will allow us to compare changes over time (within-group changes) and differences between groups simultaneously. Covariates (e.g., length of enteral and solid feeding and length of stay in acute and rehabilitation care) will be accounted for in these analyses. Overall cross-sectional between-group comparisons, such as baseline comparisons used to examine pre-test parameters, may be performed using the two-group t-test, and overall within-group comparisons may be performed using the paired t-test. If assumptions of normality of distribution for the above tests are not tenable, variables may be transformed prior to analysis or appropriate nonparametric tests, such as the Wilcoxon rank-sum and signed-rank tests, may be used. Statistical tests will be twosided and will be performed using a $5 \%$ significance level. Stratified analyses accounting for different injury levels will also be performed. These analyses will be specific to the distribution of data in each individual subgroup, which will minimize heterogeneity and allow us to analyze subgroups that may be more statistically homogenous and clinically relevant. SAS software, version 9.4 or later, will be used to conduct the statistical analyses. Multiple imputation methods will be used to address missing data for variables with moderate amounts of missing data ( $\geq 10 \%)$ and after examining whether data are missing completely at random (MCAR), at random (MAR), or not at random (MNAR). Missing data analysis will not be performed on variables with $<10 \%$ missing data to avoid statistical bias larger than that obtained through the use of complete case analysis.

For microbiome analysis, alpha diversity indices will be analyzed using linear mixed-model with time (pre and post) and group (KD and SD) as fixed factors and participant as a random effect. Permutational multivariate analysis of variance (PERMANOVA) testing will be used to assess the effects of time, 
treatment, and time $X$ treatment on beta diversity indices. The principal coordinate analysis will be performed to visualize the beta diversity distance matrices (18).

For targeted proteomics, three specific analytic approaches will be utilized. First, two-sample t-tests, or Wilcoxon rank-sum tests if the normality assumption is questionable, will be used to examine differences in the number of selected peptides and proteins between diet groups at discharge (approximately 5 weeks). Because of the number of proteins to be examined, a false discovery rate of 0.2 will be used to account for multiple hypothesis tests. The second approach will examine the manner in which levels of selected peptides vary across the longitudinal time points of baseline, rehabilitation, and discharge. To examine these patterns, mixed linear models such as repeated measures models using time as a withinperson factor and diet as a between-person factor will be constructed. Finally, Pearson's correlation will be used to measure the strength and direction of linear associations between selected peptide levels and 5week outcomes of safety, neurological, functional, and glycemic levels. Stratified analyses accounting for different injury levels will also be performed. These analyses will be specific to the distribution of data in each individual subgroup, which will minimize heterogeneity and allow us to analyze subgroups that may be more statistically homogenous and clinically relevant. SAS software, version 9.4 or later, will be used to conduct the statistical analyses.

\section{Trial monitoring}

No regular external trial auditing is scheduled. However, the Data and Safety Monitoring Committee (DSMC) includes 2 external safety monitors and 3 study personnel including the principal investigator, study physician, and a post-doctoral fellow. Following key investigators and study staff provide DSMC with information regarding safety endpoints, data quality and validity quarterly over the course of the study. All human participant data, ranging from recruitment/screening to diet intervention and testing to laboratory tests, are reviewed quarterly (every 3 months) attended by key study staff, the PI, and the DSMC committee. This includes updates on any new hazards, risks or adverse events, and plans of action. Additional investigators and staff will be asked to participate as the need for their input or expertise arises. If during the course of these meetings, particular unforeseen hazards or risks are identified that may predispose to an unusually high number of serious adverse events, the PI consults the appropriate members of the investigative team, as well as the UAB IRB and NIH/NINR to determine if the study should be terminated or altered in some way. Any procedure that is deemed hazardous is eliminated from the study and replaced with an alternative if one with reasonable risk can be identified.

\section{Adverse event monitoring and reporting}

Reporting. The principal investigator will be responsible for the accurate documentation, investigation, and follow-up of all possible study-related adverse events. All adverse events are reported by the PI to the UAB IRB with a description of the event, when and how it was reported, and appropriate documentation to corroborate the event. The description of the adverse event includes all information listed on the case report forms. The IRB then determines whether additional reporting to the NIH/NINR is required. 
- Serious Adverse Events. All serious adverse events are reported immediately to both the study physician and $\mathrm{Pl}$, and then, in turn, reported to the IRB within 48 hours. In addition, all serious adverse events that are possibly related to the study intervention are reported to the NIH/NINR within two weeks.

- Non-Serious Adverse Events. Non-serious events are reported to the IRB within five business days of the event.

Annual Reports. An annual statement summarizing the results of safety monitoring is sent to the UAB IRB and to the NIH/NINR. The annual report includes written summaries from quarterly meetings between the DSMC, key personnel and staff, descriptions of all adverse events, and a summary discussing:

(1) Whether all participants met entry criteria;

(2) Whether adverse event rates are consistent with expected rates and seriousness;

(3) Reasons for dropouts from the study;

(4) Whether continuation of the study is justified; and

(5) Whether conditions have been met for terminating the study prematurely.

\section{Protocol Amendments}

Any change to the protocol requires a written protocol amendment that must be approved by $\mathrm{NIH} / \mathrm{NINR}$ and IRB before implementation. Upon acceptance from the sponsor and IRB, the PI makes updates and edits to the study record published on ClinicalTrials.gov. If the PI determines that an immediate change or deviation from the protocol is necessary for safety reasons to eliminate an immediate hazard to the participants the IRB will be notified immediately.

\section{Confidentiality}

To protect privacy and confidentiality, any original paperwork documenting a participant's name and PHI is stored in a locked cabinet in the research coordinator's office, while any digital study records involving $\mathrm{PHI}$ are stored in REDCap and/or on computers requiring password authentication that are stored in locked offices and are behind secure firewalls. Records that identify study participants are kept confidential as required by law, and every effort is made to maintain the confidentiality of participants' study records. Except when required by law or if necessary to protect their rights or welfare, study participants are not identified by name or any other identifying characteristic in records disclosed to those outside of the study staff.

\section{Access to data}

Only study staff, the UAB IRB, official overseers of clinical research at UAB, and representatives of the NIH have access to study records, data, and specimens; all access is on a need-to-know basis. All study staff are trained in HIPAA standards for protecting $\mathrm{PHI}$ and do not refer to $\mathrm{PHI}$ or confidential information in the 
presence of individuals outside of the study team. Moreover, each study staff member's access to participants' data is limited to only the functions for which s/he is responsible.

\section{Dissemination Policy}

The results of our research will be disseminated to three major stakeholders: (1) the scientific community; (2) individuals with spinal cord injury (SCl); and (3) the general public.

\section{A. Scientific Community}

Timeline: Up to 18 months after the last participant completes the study intervention.

The results of this project will be presented at scientific conferences, including at the American Congress of Rehabilitation Medicine (ACRM), the American Spinal Injury Association, and the Society for Neuroscience annual meetings. In addition, results will be published in peer-reviewed journals with open access policies and/or in top-tier journals.

\section{B. Individuals with SCI}

Timeline: Up to 12 months after the last participant completes the study intervention.

- The UAB Spinal Cord Injury Model System Information Network Website. This website, which averages more than 43,000 visits per year, is newly designed and features a comprehensive collection of links (over 360 to date) to SCl-related information provided by reputable organizations, associations and educational institutions, including ones from the Model Systems Knowledge Translation Center (MSKTC) and other SCIMS centers. The site will be regularly updated with nutrition information and links to other reliable sites of interest and value to $\mathrm{SCl}$ consumers. All educational materials written and produced from this project will be made available free on this website.

- Pushin' On. The UAB-SCIMS newsletter (now a fully digital newsletter) has been published for 33 years. It provides persons with $\mathrm{SCl}$ and their families with information of interest. Over the years, the newsletter has featured original articles along with news, information, and synopses of research of importance to individuals with SCl. The study team will work with the newsletter editor to write a synopsis of the proposed research trial, including a description of the diet-intervention and a lay summary of major findings and how to implement them.

- Paraplegia News (PN). For more than 60 years, PN has been a leader in the wheelchair community of pertinent practical news and information. Paraplegia News Online is an extension of PN easily accessible on the world-wide-web and is dedicated to bringing the very best of real-time, up to the moment news and information for wheelchair users, family members, and medical professionals on the go. The study team will work with the newsletter editor to write a synopsis of proposed research trial including the description of diet-intervention and a lay summary of major findings and how to implement them.

\section{General Public}


Timeline: Up to 18 months after the last participant completes the study intervention.

Study findings will be communicated to the general public by collaborating with journalists. The principal investigator will reach out to several of her contacts in radio, TV, and print media to plan to write a feature article on the proposed project.

\section{Discussion}

Despite extensive research, no pharmacologic therapy has demonstrated significant improvement effects in the neurological or functional recovery in people with $\mathrm{SCl}$, and the need for innovative therapies remains urgent. The scientific premise of this project rests upon accumulating evidence that diet-based therapies, such as the KD, offer effective neuroprotection against secondary injury cascades and improve forelimb motor function in a rat model of SCl and improve upper extremity motor function in patients with acute SCl. The KD is a high-fat, low-carbohydrate diet designed to mimic the metabolic and biochemical changes that occur during calorie restriction, specifically ketosis. Ketone bodies have been shown to exert neuroprotective effects by preventing oxidative damage; attenuating neuroinflammation and glutamate excitotoxicity, and inhibiting apoptosis in the brain and spinal cord. Because glutamate excitotoxicity, inflammation, and induction of apoptotic pathways lead to progressive degeneration in the spinal cord shortly after the injury, inhibition of these processes by ketone bodies may enhance neurological recovery after an SCl. In support of these hypotheses, we recently showed, for the first time, that compared with an $\mathrm{SD}$, five weeks of KD improved upper extremity motor function in patients with acute $\mathrm{SCl}(\mathrm{Fig} .1)(20)$. In addition, a neuroinflammatory blood protein, fibrinogen, was present at lower levels in the KD serum samples than in the SD serum samples. Taken together, our preliminary results suggest that a KD may induce anti-inflammatory effects in part by reducing fibrinogen, which promotes neuroprotection and improved recovery.

On the basis of this preliminary data in patients, we have designed a randomized, controlled trial to examine the effects of a novel nutritional intervention, the ketogenic diet, on improving sensory and motor recovery, functional independence, and glycemic control in patients with acute $\mathrm{SCl}$ and on the molecular mechanisms thereof. Our findings may lead to a safe, effective, simple, and economical intervention that improves health outcomes for these patients. Changing patients to a more neuroprotective diet during acute care of $\mathrm{SCl}$ can be implemented anywhere in the world at low cost and without major regulatory hurdles. Better functional recovery will lead to a better quality of life and longterm health outcomes in individuals with SCl. Therefore, the risks incurred by participants in the study are minor relative to the benefit of improving the quality of life of individuals with $\mathrm{SCl}$.

\section{Trial status}

The study has been active and open for enrollment since September 2019. Enrollment is expected to be completed in December 2023. The clinical trial number is NCT03509571. The protocol version is version 2, January 2nd, 2020. 


\section{Abbreviations}

SCI:Spinal Cord Injury; KD:Ketogenic Diet; SD:Standard Hospital Diet; ISNCSCI:Neurological examination via International Standards for Neurological Classification of SCl; AIS:American Spinal Injury Association Impairment Scale; EPT; Electrical Perception Thresholds; SCIM:Spinal Cord Independence Measure; REE:Resting Energy Expenditure; UAB:University of Alabama at Birmingham; SRC:Spain Rehabilitation Center; CCTS:UAB Center for Clinical and Translational Science; EMRA:UAB Emergency Medicine Research Assistance; HDL:High-Density Lipoprotein; LDL:Low-Density Lipoprotein; ALT:Alanine Aminotransferase AST:Aspartate Transaminase; ALP:Alkaline Phosphatase; NINDS:National Institute of Neurological Disorders and Stroke; CDE:Common Data Elements; ASIA:American Spinal Injury Association; QIIME:Quantitative Insights into Microbial Ecology; OUT:Operational Taxonomy Units; MS:Mass Spectrometry; ESI:Electrospray Ionization; IRB:Institutional Review Board; ANCOVA:Analysis of Covariance; MCAR:Missing Completely at Random; PERMANOVA:Permutational Multivariate Analysis of Variance; DSMC:The Data and Safety Monitoring Committee; ACRM:American Congress of Rehabilitation Medicine; MSKTC:Model Systems Knowledge Translation Center; PN:Paraplegia News.

\section{Declarations}

Authors Contribution: $A D, J L$, and $C Y-F$ wrote the manuscript. $A D, J L, C M, S B, B G, K K, C Y-F$ all contribute to the design, planning, and/or implementation of the protocol. All authors read and approved the final manuscript.

Funding: This work was supported by the National Institute of Nursing Research grant \#1R01NR01644301A1 (Dr. Lois Tully, 301-594-5968). The funding agency is not involved with study design, collection, management, analysis, or interpretation of data, nor writing of the report or the decision to submit the report for publication.

Availability of data and materials: The datasets used and/or analyzed during the current study will be available from the corresponding author on reasonable request.

Ethics approval and consent to participate: This study was approved by the UAB IRB (IRB-F151211001) prior to any participants enrolling. All participation is on a voluntary basis, and each participant is required to provide informed consent and sign a HIPAA authorization form prior to their participation in the study protocol. Participants are given options to provide consent for collection and use of their data and specimens in ancillary studies.

Consent for publication: Not applicable.

Competing interests: The authors declare that they have no competing interests.

\section{References}


1. Bauman WA, Spungen AM. Disorders of carbohydrate and lipid metabolism in veterans with paraplegia or quadriplegia: a model of premature aging. Metab Clin Exp. 1994;43(6):749-56.

2. Bauman WA, Spungen AM. Invited review carbohydrate and lipid metabolism in chronic spinal cord injury. J Spinal Cord Med 2001;24(4):266-77.

3. Jensen M, Molton I, Groah S, Campbell M, Charlifue S, Chiodo A, et al. Secondary health conditions in individuals aging with SCl: terminology, concepts and analytic approaches. Spinal cord. 2012;50(5):373.

4. Strauss DJ, DeVivo MJ, Paculdo DR, Shavelle RM. Trends in life expectancy after spinal cord injury. Arch Phys Med Rehabil. 2006;87(8):1079-85.

5. Michael J, Krause JS, Lammertse DP. Recent trends in mortality and causes of death among persons with spinal cord injury. Arch Phys Med Rehabil. 1999;80(11):1411-9.

6. Shavelle RM, DeVivo MJ, Paculdo DR, Vogel LC, Strauss DJ. Long-term survival after childhood spinal cord injury. J Spinal Cord Med. 2007;30(sup1):S48-S54.

7. Silva NA, Sousa N, Reis RL, Salgado AJ. From basics to clinical: a comprehensive review on spinal cord injury. Prog Neurobiol. 2014;114:25-57.

8. Park E, Velumian AA, Fehlings MG. The role of excitotoxicity in secondary mechanisms of spinal cord injury: a review with an emphasis on the implications for white matter degeneration. J Neurotrauma. 2004;21(6):754-74.

9. Badhiwala JH, Wilson JR, Kwon BK, Casha S, Fehlings MG. A review of clinical trials in spinal cord injury including biomarkers. J Neurotrauma 2018;35(16):1906-17.

10. Kobayakawa K, Kumamaru H, Saiwai H, Kubota K, Ohkawa Y, Kishimoto J, et al. Acute hyperglycemia impairs functional improvement after spinal cord injury in mice and humans. Sci Transl Med 2014;6(256):256ra137-256ra137.

11. Sala F, Menna G, Bricolo A, Young W. Role of glycemia in acute spinal cord injury: data from a rat experimental model and clinical experience. Ann N Y Acad Sci 1999;890(1):133-54.

12. Freeman JM, Vining EP, Pillas DJ, Pyzik PL, Casey JC. The efficacy of the ketogenic diet-1998: a prospective evaluation of intervention in 150 children. Pediatrics. 1998;102(6):1358-63.

13. Vanltallie TB, Nonas C, Di Rocco A, Boyar K, Hyams K, Heymsfield S. Treatment of Parkinson disease with diet-induced hyperketonemia: a feasibility study. Neurology. 2005;64(4):728-30.

14. Gasior M, Rogawski MA, Hartman AL. Neuroprotective and disease-modifying effects of the ketogenic diet. Behav Pharmacol. 2006;17(5-6):431.

15. Prins ML, Matsumoto JH. The collective therapeutic potential of cerebral ketone metabolism in traumatic brain injury. J Lipid Res.2014;55(12):2450-7.

16. Reger MA, Henderson ST, Hale C, Cholerton B, Baker LD, Watson G, et al. Effects of $\beta$-hydroxybutyrate on cognition in memory-impaired adults. Neurobiol Aging. 2004;25(3):311-4.

17. Hussain TA, Mathew TC, Dashti AA, Asfar S, Al-Zaid N, Dashti HM. Effect of low-calorie versus lowcarbohydrate ketogenic diet in type 2 diabetes. Nutrition. 2012;28(10):1016-21. 
18. Feinman RD, Pogozelski WK, Astrup A, Bernstein RK, Fine EJ, Westman EC, et al. Dietary carbohydrate restriction as the first approach in diabetes management: critical review and evidence base. Nutrition. 2015;31(1):1-13.

19. Streijger F, Plunet WT, Lee JH, Liu J, Lam CK, Park S, et al. Ketogenic diet improves forelimb motor function after spinal cord injury in rodents. PLoS One. 2013;8(11):e78765.

20. Yarar-Fisher C, Kulkarni A, Li J, Farley P, Renfro C, Aslam H, et al. Evaluation of a ketogenic diet for improvement of neurological recovery in individuals with acute spinal cord injury: a pilot, randomized safety and feasibility trial. Spinal Cord Ser Cases. 2018;4(1):88.

21. Bough KJ, Wetherington J, Hassel B, Pare JF, Gawryluk JW, Greene JG, et al. Mitochondrial biogenesis in the anticonvulsant mechanism of the ketogenic diet. Ann Neurol 2006;60(2):223-35.

22. Veech RL, Chance B, Kashiwaya Y, Lardy HA, Cahill Jr GF. Ketone bodies, potential therapeutic uses. IUBMB life. 2001;51(4):241-7.

23. Kramer JK, Taylor P, Steeves JD, Curt A. Dermatomal somatosensory evoked potentials and electrical perception thresholds during recovery from cervical spinal cord injury. Neurorehabil Neural Repair. 2010;24(4):309-17.

24. Catz A, Itzkovich M. Spinal Cord Independence Measure: comprehensive ability rating scale for the spinal cord lesion patient. J Rehabil Res Dev 2007;44(1):65.

25. Catz MI, Flavia Steinberg, Ora Philo, Haim Ring, Jacob Ronen, Raluca Spasser, Reuven Gepstein, Ada Tamir, Amiram. The Catz-Itzkovich SCIM: a revised version of the spinal cord independence measure. Disabil Rehabil. 2001;23(6):263-8.

26. Pouw M, Van Middendorp J, van Kampen A, Curt A, van de Meent H, Hosman A. Diagnostic criteria of traumatic central cord syndrome. Part 3: descriptive analyses of neurological and functional outcomes in a prospective cohort of traumatic motor incomplete tetraplegics. Spinal Cord. 2011;49(5):614.

27. Spinal Cord Injury Facts and Figures at a Glance 20182018 [Available from: https://www.nscisc.uab.edu/Public/Facts\%20and\%20Figures\%20-\%202018.pdf.

28. DeVivo M, Biering-Sørensen F, New P, Chen Y. Standardization of data analysis and reporting of results from the International Spinal Cord Injury Core Data Set. Spinal cord. 2011;49(5):596.

29. Ditunno J, Young W, Donovan W, Creasey G. The international standards booklet for neurological and functional classification of spinal cord injury. Spinal Cord. 1994;32(2):70.

30. Maynard Jr FM, Bracken MB, Creasey G, Ditunno Jr JF, Donovan WH, Ducker TB, et al. International standards for neurological and functional classification of spinal cord injury. Spinal cord. 1997;35(5):266.

31. Waters RL, Adkins RH, Yakura JS, Sie I. Motor and sensory recovery following complete tetraplegia. Arch Phys Med Rehabil. 1993;74(3):242-7.

32. Björhall K, Miliotis T, Davidsson P. Comparison of different depletion strategies for improved resolution in proteomic analysis of human serum samples. Proteomics. 2005;5(1):307-17. 
33. Sharma V, Eckels J, Schilling B, Ludwig C, Jaffe JD, MacCoss MJ, et al. Panorama Public: A public repository for quantitative data sets processed in Skyline. Mol Cell Proteomics. 2018;17(6):1239-44.

34. LeBlanc A, Michaud SA, Percy AJ, Hardie DB, Yang J, Sinclair NJ, et al. Multiplexed MRM-based protein quantitation using two different stable isotope-labeled peptide isotopologues for calibration. J Proteome Res. 2017;16(7):2527-36.

35. Sajic T, Liu Y, Aebersold R. Using data-independent, high-resolution mass spectrometry in protein biomarker research: perspectives and clinical applications. Proteomics Clin Appl.2015;9(3-4):307-21.

36. Kramer JL, Lammertse DP, Schubert M, Curt A, Steeves JD. Relationship between motor recovery and independence after sensorimotor-complete cervical spinal cord injury. Neurorehabil Neural Repair. 2012;26(9):1064-71.

\section{Supplementary File Legend}

Additional File 1: SPIRIT checklist

\section{Figures}

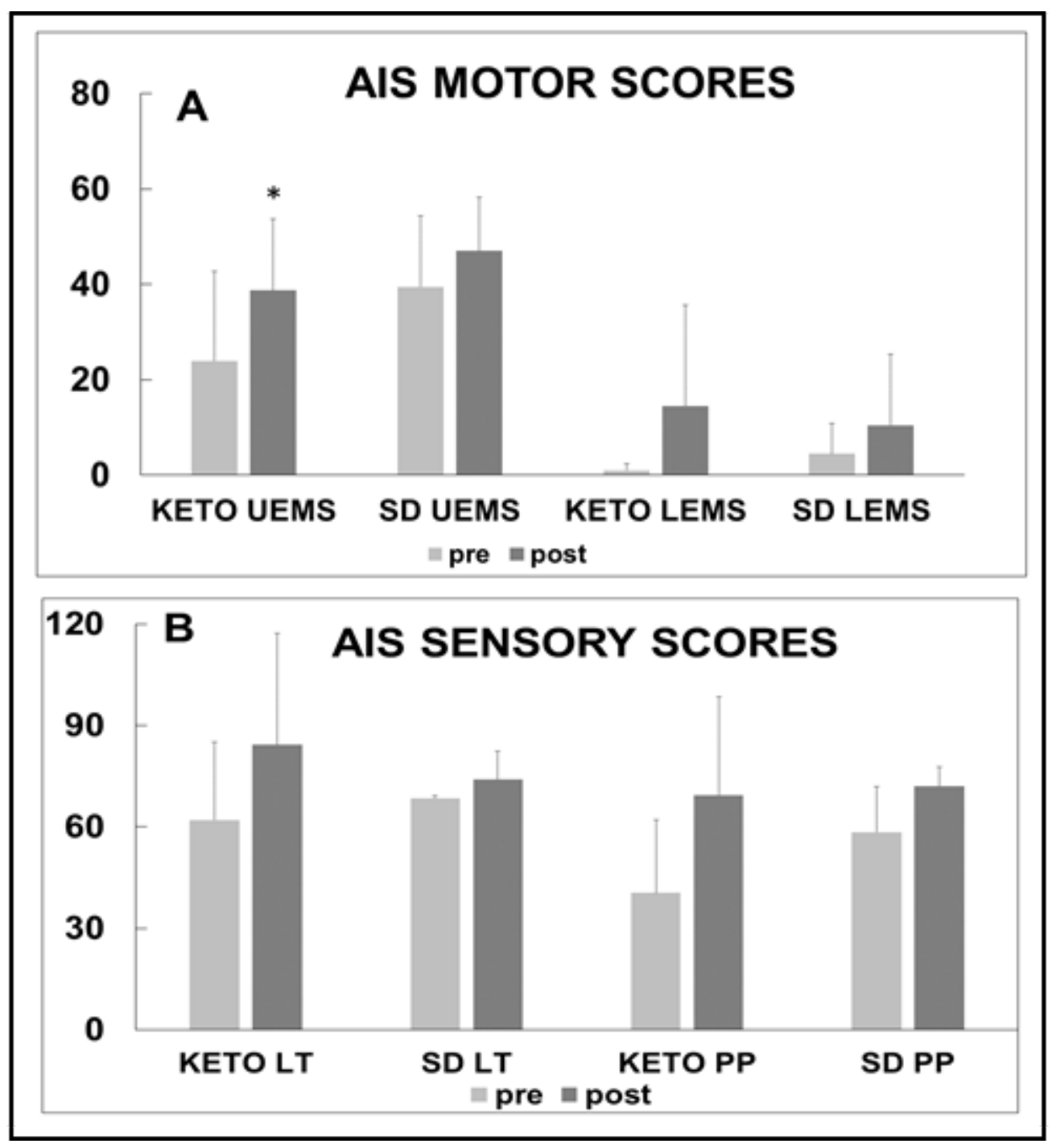


Figure 1

Effects of the ketogenic diet (KD) vs. standard (SD) on motor and sensory scores in patients with spinal cord injury (SCI). (A): American Spinal Injury Association (ASIA) Impairment Scale (AIS) upper extremity motor scores (UEMS) and lower extremity motor scores (LEMS)(B): AIS Light-touch (LT) and pin-prick (PP) sensory scores.

\begin{tabular}{|c|c|c|c|c|c|c|c|c|c|}
\hline & \multicolumn{9}{|c|}{ Study Period } \\
\hline & \multirow{2}{*}{$\begin{array}{c}\text { Enrollment* }^{\star} \\
\text { Within } 72 \\
\text { hours after } \\
\text { injury }\end{array}$} & \multirow{2}{*}{$\begin{array}{c}\text { Allocation* }^{*} \\
0\end{array}$} & \multicolumn{7}{|c|}{ Post-allocation } \\
\hline TIMEPOINTS & & & Baseline* $^{\star}$ & Wk1 & Wk2 & Wk3 & Wk4 & Wk5 & Post $^{\star}$ \\
\hline \multicolumn{10}{|l|}{ ENROLLMENT: } \\
\hline Eligibility screen & $\mathrm{X}$ & & & & & & & & \\
\hline Informed consent & $\mathrm{X}$ & & & & & & & & \\
\hline Allocation & & $\mathrm{X}$ & & & & & & & \\
\hline \multicolumn{10}{|l|}{ INTERVENTIONS: } \\
\hline \multicolumn{10}{|l|}{ [Ketogenic diet group] } \\
\hline \multicolumn{10}{|l|}{ [Standard hospital diet group] } \\
\hline \multicolumn{10}{|l|}{ ASSESSMENTS: } \\
\hline [Resting Energy Expenditure] & & & $\mathrm{X}$ & $\mathrm{X}$ & & & & & \\
\hline [C-reactive protein] & & & $\mathrm{X}$ & $\mathrm{X}$ & $\mathrm{X}$ & $\mathrm{X}$ & $\mathrm{X}$ & $\mathrm{X}$ & $\mathrm{X}$ \\
\hline [Lipid profile $\left.{ }^{*}\right]$ & & & $\mathrm{X}$ & $\mathrm{X}$ & $\mathrm{X}$ & $\mathrm{X}$ & $\mathrm{X}$ & $\mathrm{X}$ & $\mathrm{X}$ \\
\hline [Liver function $\left.{ }^{\&}\right]$ & & & $\mathrm{X}$ & $\mathrm{X}$ & $\mathrm{X}$ & $\mathrm{X}$ & $\mathrm{X}$ & $\mathrm{X}$ & $\mathrm{X}$ \\
\hline [Beta-hydroxybutyrate] & & & $\mathrm{X}$ & $\mathrm{X}$ & $\mathrm{X}$ & $\mathrm{X}$ & $\mathrm{X}$ & $\mathrm{X}$ & $\mathrm{X}$ \\
\hline [AIS motor score] & & & $\mathrm{X}$ & & & $\mathrm{X}$ & & $\mathrm{X}$ & $\mathrm{X}$ \\
\hline [AIS sensory score] & & & $\mathrm{X}$ & & & $\mathrm{X}$ & & $\mathrm{X}$ & $\mathrm{X}$ \\
\hline [Electrical Perception Threshold] & & & $\mathrm{X}$ & & & & & $\mathrm{X}$ & $\mathrm{X}$ \\
\hline $\begin{array}{r}\text { [Spinal cord independence } \\
\text { measure] }\end{array}$ & & & $\mathrm{X}$ & & & $X$ & & $X$ & $x$ \\
\hline [Glucose] & & & $\mathrm{X}$ & & & $\mathrm{X}$ & & $\mathrm{X}$ & $\mathrm{X}$ \\
\hline [Insulin] & & & $\mathrm{X}$ & & & $\mathrm{X}$ & & $\mathrm{X}$ & $\mathrm{X}$ \\
\hline [Urine collection] & & & $\mathrm{X}$ & $\mathrm{X}$ & $\mathrm{X}$ & $\mathrm{X}$ & $\mathrm{X}$ & $\mathrm{X}$ & $\mathrm{X}$ \\
\hline [Stool collection] & & & $\mathrm{X}$ & $\mathrm{X}$ & $\mathrm{X}$ & $\mathrm{X}$ & $\mathrm{X}$ & $\mathrm{X}$ & $\mathrm{X}$ \\
\hline [Protein analyses $\left.{ }^{s}\right]$ & & & $\mathrm{X}$ & & & $\mathrm{X}$ & & $\mathrm{X}$ & $\mathrm{X}$ \\
\hline $\begin{array}{l}\text { Figure 2. Standard Protocol Items: Recommenda } \\
\text { Wk: week AIS: American Spinal Injury Associatio } \\
\text { "Allocation and baseline testing occur within } 72 \mathrm{~h} \\
\text { " Including Cholesterol, Triglycerides, HDL-chole } \\
\text { \&As reflected by liver enzymes ALT, ASP, ALP, a } \\
\text { \$Serum fibrinogen, extracellular signal-regulated }\end{array}$ & $\begin{array}{l}\text { ons for Interventiona } \\
\text { Impairment Scale } \\
\text { urs of injury; post-int } \\
\text { erol, calculated LDL } \\
\text { umin, bilirubin and t }\end{array}$ & $\begin{array}{l}\text { Trials (SPIRIT) sch } \\
\text { rvention assessme } \\
\text { holesterol } \\
\text { tal protein. } \\
18 \text { integrin receptor }\end{array}$ & ints are performe & d prior to & each pa & tient's di & scharge. & & \\
\hline
\end{tabular}

Figure 2

Standard Protocol Items: Recommendations for Interventional Trials (SPIRIT) schedule Wk: week AIS: American Spinal Injury Association Impairment Scale *Allocation and baseline testing occurs within 72 hours of injury; post-intervention assessments are performed prior to each patient’s discharge. $¥$ Including Cholesterol, Triglycerides, HDL-cholesterol, calculated LDL-cholesterol \&As reflected by liver enzymes alanine Aminotransferase (ALT), aspartate aminotransferase (AST), alkaline phosphatase (ALP), albumin, bilirubin and total protein. 


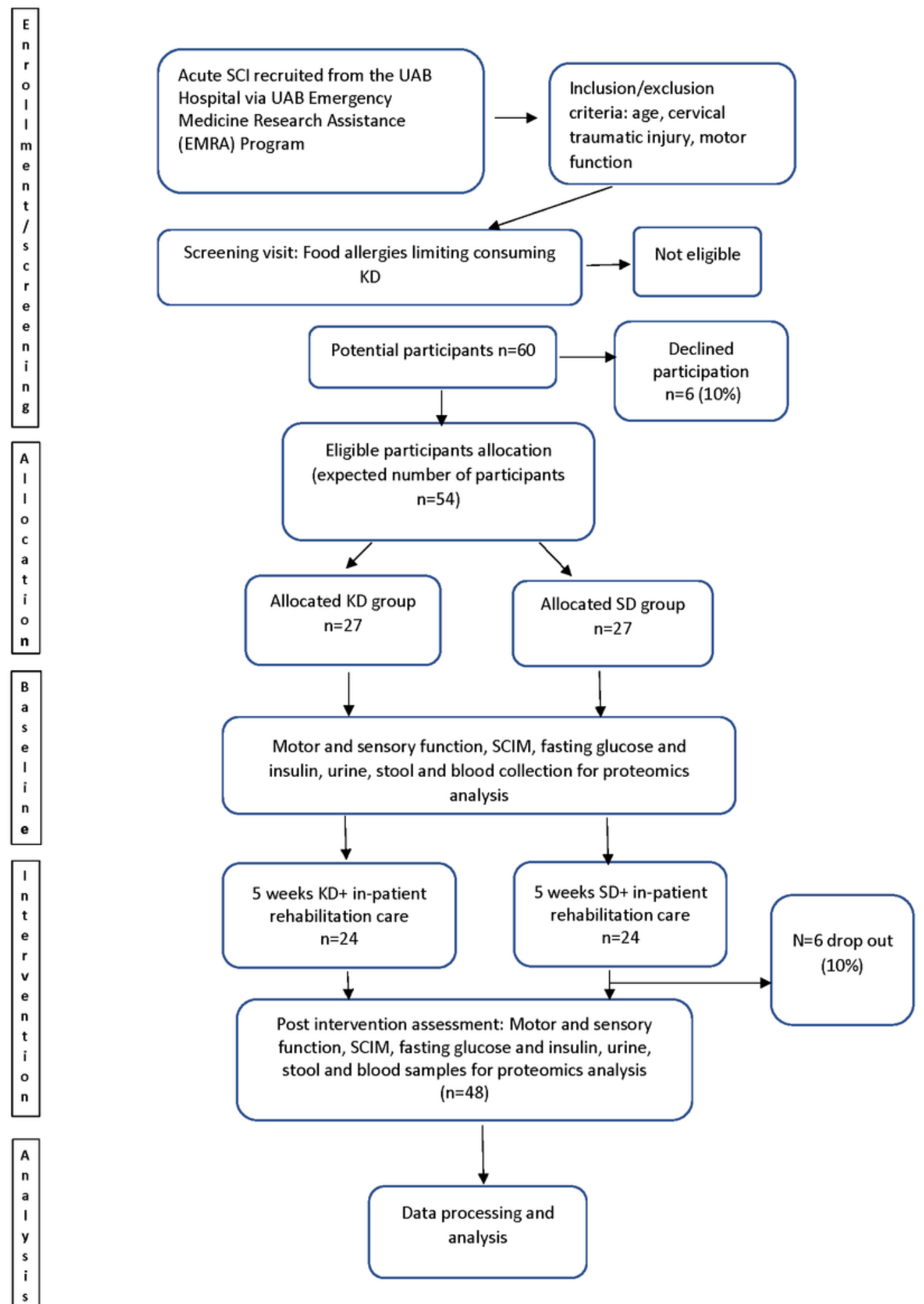

Figure 3

Flow diagram KD: ketogenic diet; SD: standard hospital diet; SCIM: spinal cord independent measurement

\section{Supplementary Files}

This is a list of supplementary files associated with this preprint. Click to download. 
- additionalfile1SPIRITchecklist14.01.2020.doc 